\title{
TOXICIDADE DE ACARICIDAS A OVOS E ADULTOS DE Ceraeochrysa cubana (Hagen, 1861) (Neuroptera: Chrysopidae)
}

\author{
Toxicity of acaricides to eggs and adults of Ceraeochrysa cubana \\ (Hagen, 1861) (Neuroptera: Chrysopidae)
}

\author{
Geraldo Andrade Carvalho', César Freire Carvalho², Márcio do Nascimento Ferreira³
}

\begin{abstract}
RESUMO
Objetivou-se, neste trabalho, avaliar o efeito de treze acaricidas sobre ovos e adultos de Ceraeochrysa cubana (Hagen, 1861). Os acaricidas foram pulverizados por meio de torre de Potter, nas concentrações recomendadas para o controle de ácaros na cultura dos citros. Os ensaios foram conduzidos sob condições controladas de $25 \pm 2^{\circ} \mathrm{C}$, umidade relativa de $70 \pm 10 \%$ e fotofase de 12 horas. Os acaricidas não foram tóxicos ao predador em sua fase de ovo. Em relação aos adultos, o carbosulfan e o triazophos foram altamente tóxicos (100\% de mortalidade), enquanto que os piretróides bifenthrin e fenpropathrin causaram mortalidade aproximadamente de $60 \%$. Os acaricidas dicofol, bromopropylate, tetradifon, clofentezine, abamectin, hexythiazox, cyhexatin e óxido de fenbutatin não provocaram mortalidade dos adultos de C. cubana. Flufenoxuron inibiu a capacidade de oviposição e provocou a inviabilização dos ovos. Baseando-se na escala da IOBC, os acaricidas carbosulfan, triazophos e flufenoxuron foram enquadrados na classe $4=$ nocivos; bifenthrin e fenpropathrin na classe 2 = levemente nocivos, e dicofol, bromopropylate, tetradifon, clofentezine, abamectin, hexythiazox, cyhexatin e óxido de fenbutatin na classe $1=$ inócuos a $C$. cubana. Os compostos enquadrados na classe 1 podem ser recomendados em programas de manejo integrado de pragas de citros objetivando a integração entre os métodos químico e biológico por meio dessa espécie de predador.
\end{abstract}

Termos para indexação: Citros, pesticidas, crisopídeo, predador, seletividade.

\begin{abstract}
The objective of this work was to evaluate the effects of thirteen acaricides on eggs and adults of the lacewing Ceraeochrysa cubana (Hagen, 1861). The acaricides were sprayed using a Potter's tower at the rate recommended for mite control in citrus orchard. The trials were performed in climatic chamber at $25 \pm 2^{\circ} \mathrm{C}, 70 \pm 10 \% \mathrm{RH}$ and photophase of 12 hours. The egg stage showed high tolerance to the tested acaricides. Carbosulfan and triazophos caused 100\% adult mortality, whereas bifenthrin and fenpropathrin caused around $60 \%$. All other acaricides, dicofol, bromopropylate, tetradifon, clofentezine, abamectin, hexythiazox, cyhexatin and fenibutatin oxide did not cause mortality of C. cubana adult. Flufenoxuron reduced adult egg laying and all eggs were nonviable. According to IOBC scale, the acaricides carbosulfan, triazophos and flufenoxuron were classified into class $4=$ harmful; bifenthrin and fenpropathrin into class 2 = slightly toxic; and dicofol, clofentezine, abamectin, hexythiazox, cyhexatin, and fenbutatin oxide into class $1=$ harmless to $C$. cubana. The compounds classified as first class may be recommended in programs of integrated pest management of citrus aiming at the integration between the biological and chemical control through this kind of predator.
\end{abstract}

Index terms: Citrus, pesticides, green lacewing, predator, selectivity.

(Recebido em 19 de junho de 2009 e aprovado em 29 de abril de 2010)

\section{INTRODUÇÃO}

Existem vários fatores que limitam a produção de citros, sendo que insetos, ácaros e patógenos causam as maiores perdas (Parra et al., 2003). Para controlar estas pragas realizam-se aplicações de agrotóxicos, os quais podem provocar desequilíbrios biológicos, ressurgimento de pragas e, consequentemente, aparecimento de populações de pragas resistentes.

O emprego de agrotóxicos de maneira ecologicamente seletiva minimiza a exposição de inimigos naturais e, ao mesmo tempo, controla as espécies-praga, constituindo uma importante estratégia do controle químico dentro da filosofia do manejo integrado de pragas (Foerster, 2002).

Um dos principais grupos de inimigos naturais presentes em citros são os crisopídeos, que atualmente têm recebido considerável atenção de muitos pesquisadores em todo o mundo. Esses predadores exercem importante papel no controle biológico de artrópodes fitófagos, podendo ser encontrados em muitas culturas de interesse econômico, tais como os citros (Souza et al., 1996). Os crisopídeos destacam-se por sua alta capacidade reprodutiva, grande voracidade e plasticidade ecológica em agroecossistemas e por estarem associados

\footnotetext{
1Universidade Federal de Lavras/UFLA - Departamento de Entomologia/DEN - Cx. P. 3037 - 37200-000 - Lavras, MG - gacarval@ufla.br ${ }^{2}$ Universidade Federal de Lavras/UFLA - Departamento de Entomologia/DEN - Lavras, MG

${ }^{3}$ Universidade Federal do Mato Grosso/UFMT - Faculdade de Agronomia e Veterinária/FAMEV - Cuiadá, MT
} 
às mais diferentes pragas (Carvalho \& Souza, 2009). Segundo Parra et al. (2003), as espécies de crisopídeos mais encontradas em pomares de citros são Chrysoperla externa (Hagen, 1861) e Ceraeochrysa cubana (Hagen, 1861). Também, de acordo com Souza et al. (1996), estes predadores ocorrem com grande frequência em pomares cítricos na região de Lavras, podendo destacar a espécie C. cubana. As larvas desses predadores consomem pulgões, cochonilhas, moscas-brancas e ácaros, enquanto os adultos alimentam-se de pólen e solução açucarada (nectários de plantas e "honeydew" produzido pelas próprias presas homópteras).

A conservação de crisopídeos em pomares de citros deve ser considerada ao se estabelecer um programa de manejo de pragas e isso dependerá da compatibilidade com os outros métodos de controle, especialmente o químico. A maioria dos agrotóxicos que são aplicados em pomares cítricos para o controle de pragas, geralmente, causa efeitos indesejáveis sobre os inimigos naturais (Gravena, 1990). Assim, objetivou-se, com o presente trabalho, avaliar os efeitos de alguns acaricidas utilizados em pomares cítricos sobre ovos e adultos de C. cubana em laboratório, visando obter informações que colaborem para o melhor manejo de pragas nessa cultura.

\section{MATERIAL E MÉTODOS}

Os bioensaios foram realizados sob condições de $25 \pm 2{ }^{\circ} \mathrm{C}$, umidade relativa de $70 \pm 10 \%$ e fotofase de 12 horas.
Os predadores utilizados estavam entre a $2^{\underline{a}}$ e $7^{a}$ geração de criação em laboratório, sendo que a partir da $4^{\mathrm{a}}$ geração introduziram-se novos insetos coletados em pomar de citros a fim de se evitar uma possível degeneração da população.

Os acaricidas foram diluídos em água destilada, utilizando-se as concentrações recomendadas pelos respectivos fabricantes para o controle de ácaros na cultura de citros (Tabela 1). No tratamento testemunha utilizou-se somente água destilada.

Efeitos dos acaricidas sobre ovos de C. cubana. Para a obtenção dos ovos, 10 fêmeas de $C$. cubana da criação de laboratório foram mantidas, durante 24 horas, em gaiola de PVC de $15 \mathrm{~cm}$ de diâmetro x $20 \mathrm{~cm}$ de altura revestida internamente com papel-filtro branco no qual as fêmeas ovipositaram. Após este período, o papel-filtro foi retirado e dividido em várias partes retangulares, de modo que cada retângulo tivesse, no mínimo, 10 ovos. Esses retângulos contendo os ovos foram pulverizados em torre de Potter. O volume de aplicação utilizado foi de $0,006 \mathrm{ml}$ de calda/ $\mathrm{cm}^{2}$, determinado após a calibração do equipamento de pulverização, que funcionou durante 25 segundos através de compressor regulado a uma pressão de $1,4 \mathrm{kgf} / \mathrm{cm}^{2}$.

Os ovos foram individualizados em tubos de vidro de $2 \mathrm{~cm}$ de diâmetro $\mathrm{x} 8 \mathrm{~cm}$ de altura após retirado o excesso de umidade dos retângulos de papel mediante escorrimento em ambiente arejado. Cada grupo de 10 tubos contendo ovos constituiu uma repetição. Avaliaram-se o efeito dos acaricidas sobre o período embrionário e a eclosão de larvas

Tabela 1 - Especificações e dosagens dos acaricidas utilizados nos testes de seletividade para ovos e adultos de Ceraeochrysa cubana (Hagen, 1861).

\begin{tabular}{lccc}
\hline Nome Comum & Nome Comercial & Formulação & Dosagem (g i.a./L d'água) \\
\hline Dicofol & Kelthane CE & $185 \mathrm{~g} / \mathrm{L}$ & 0,370 \\
Fenpropathrin & Meothrin CE & $300 \mathrm{~g} / \mathrm{L}$ & 0,120 \\
Bifenthrin & Talstar 100 CE & $100 \mathrm{~g} / \mathrm{L}$ & 0,020 \\
Bromopropylate & Neoron 500 CE & $500 \mathrm{~g} / \mathrm{L}$ & 0,325 \\
Triazophos & Hostathion 400 CE & $400 \mathrm{~g} / \mathrm{L}$ & 0,600 \\
Tetradifon & Tedion $80 \mathrm{CE}$ & $80 \mathrm{~g} / \mathrm{L}$ & 0,240 \\
Clofentezine & Acaristop 500 SC & $500 \mathrm{~g} / \mathrm{L}$ & 0,300 \\
Flufenoxuron & Cascade CE & $100 \mathrm{~g} / \mathrm{L}$ & 0,100 \\
Carbosulfan & Marshal 250 CE & $250 \mathrm{~g} / \mathrm{L}$ & 0,100 \\
Abamectin & Vertimec $18 \mathrm{CE}$ & $18 \mathrm{~g} / \mathrm{L}$ & 0,009 \\
Hexythiazox & Savey PM & $500 \mathrm{~g} / \mathrm{kg}$ & 0,015 \\
Cyhexatin & Hokko Cyhexatin 500 PM & $500 \mathrm{~g} / \mathrm{kg}$ & 0,250 \\
Oxido de fenbutatin & Torque 500 SC & $500 \mathrm{~g} / \mathrm{L}$ & 0,300 \\
\hline
\end{tabular}


e, posteriormente, foi estimada a porcentagem de viabilidade desses ovos.

O delineamento experimental utilizado foi inteiramente casualizado, com quatorze tratamentos (= acaricidas) e cinco repetições ( $=50$ ovos).

Efeito de acaricidas sobre adultos de $\boldsymbol{C}$. cubana. Machos e fêmeas recém-emergidos provenientes da criação de laboratório, em quantidade suficiente para a montagem do bioensaio, foram colocados juntos em gaiolas de PVC de $15 \mathrm{~cm}$ de diâmetro x $20 \mathrm{~cm}$ de altura, por um período de sete dias, para possibilitar o acasalamento. Esse período foi estabelecido com base em trabalho realizado por Venzon \& Carvalho (1992) que estudaram a biologia de C. cubana e verificaram que as fêmeas apresentaram um período médio de pré-oviposição de sete dias a partir da emergência.

Após esse período, os insetos foram separados por sexo, formando-se grupos de 10 casais por tratamento, os quais foram, então, anestesiados com $\mathrm{CO}_{2}$ durante 30 segundos para tratamento. Os grupos de insetos anestesiados foram colocados sobre papel-filtro em uma placa de Petri de $20 \mathrm{~cm}$ de diâmetro e levados à torre de Potter, onde receberam as pulverizações dos acaricidas descritos na Tabela 1 , bem como de água destilada (testemunha).

Após a aplicação dos acaricidas, os insetos foram rapidamente transferidos na proporção de um casal por gaiola de PVC de $10 \mathrm{~cm}$ de diâmetro x $10 \mathrm{~cm}$ de altura revestidas internamente com papel-filtro branco. Para a alimentação dos adultos nessas gaiolas, utilizou-se dieta composta de uma mistura de levedura de cerveja e mel, em partes iguais, adicionando-se algumas gotas de água destilada até a obtenção de uma pasta, que foi pincelada em uma tira de Parafilm ${ }^{\circledR}$, com cerca de $5 \mathrm{~cm}$ de comprimento e $1 \mathrm{~cm}$ de largura, fixando-a no interior da gaiola. $\mathrm{O}$ fornecimento de água foi feito mediante um frasco com capacidade para $19 \mathrm{ml}$, contendo um chumaço de algodão embebido em água destilada, colocado no fundo da gaiola.

O delineamento experimental utilizado foi o inteiramente casualizado com 14 tratamentos e 10 repetições, sendo que, cada gaiola contendo um casal do crisopídeo constituiu uma repetição.

Os insetos foram monitorados diariamente até a morte anotando-se as seguintes características biológicas: 1) período de oviposição - intervalo, em dias, do início ao término da oviposição; 2) período efetivo de oviposição número de dias em que as fêmeas ovipositaram; 3) capacidade diária de oviposição - relação entre o número total de ovos produzidos e o número de dias do período de oviposição; 4) capacidade total de oviposição - número total de ovos produzidos durante o período de oviposição; 5) longevidade - intervalo, em dias, da emergência à morte do adulto; 6) viabilidade de ovos - percentual de larvas eclodidas, e 7) período embrionário - intervalo, em dias, da oviposição à eclosão da larva.

O período embrionário e a viabilidade dos ovos foram determinados individualizando-se, em tubos de vidro de $6 \mathrm{ml}, 10$ ovos coletados ao acaso em cada gaiola no décimo dia após o início da oviposição e acompanhandose o desenvolvimento até a eclosão das larvas.

Análises estatísticas. A homogeneidade das variâncias foi testada pelo teste de Bartlett, indicando a necessidade de transformação dos dados para a maioria das variáveis. Para ovos, os dados de viabilidade foram transformados em $\operatorname{arc} \operatorname{sen} \sqrt{\mathrm{x}} / 100$ e os referentes ao período embrionário para $\sqrt{\mathrm{x}}$. Os relativos à produção de ovos pelas fêmeas tratadas foram transformados para $\sqrt{\mathrm{x}}$. As comparações múltiplas foram feitas pelo teste de Tukey $(\mathrm{P}<0,05)$. Todas as análises foram efetuadas utilizando-se o programa SAEG.

Além desses testes, a seletividade dos acaricidas foi comparada pelo índice de toxicidade, atribuído com base na escala proposta pela IOBC (International Organization for Biological and Integrated Control of Noxious Animals and Plants) (Hassan \& Degrande, 1996), em que a classificação é feita através do efeito total (E) de cada produto, que leva em consideração a porcentagem de mortalidade e/ou influência na reprodução do crisopídeo, sendo: classe $1=$ inócuo $(\mathrm{E}<30 \%)$, classe $2=$ levemente nocivo $(30 \% \leq \mathrm{E} \leq 79 \%)$, classe $3=$ moderadamente nocivo $(80 \% \leq \mathrm{E} \leq 99 \%)$ e classe $4=$ nocivo $(\mathrm{E}>99 \%)$.

\section{RESULTADOS E DISCUSSÃO}

Efeitos sobre a viabilidade e período embrionário de $\boldsymbol{C}$. cubana. Os ovos de C. cubana mostraram-se inócuos a todos os acaricidas estudados, nas concentrações utilizadas, apresentando médias de viabilidade que variaram de 76,8\% a $97 \%$ (Tabela 2). Os resultados desta pesquisa assemelham-se àqueles de Souza et al. (1996) para alguns dos acaricidas testados. Esses autores constataram que fenpropathrin $\left(0,09 \mathrm{~g}\right.$ i.a. $\left.\mathrm{L}^{-1}\right)$ e óxido de fenbutatin $\left(0,25 \mathrm{~g}\right.$ i.a. $\left.\mathrm{L}^{-1}\right)$, aplicados sobre os ovos desse predador, não provocaram redução do número de larvas eclodidas. São também semelhantes aos obtidos por Carvalho et al. (2002), que encontraram viabilidade de $73,3 \%$ a $90 \%$ quando aplicaram triflumuron $\left(0,0375 \mathrm{~g}\right.$ i.a. $\left.\mathrm{L}^{-1}\right)$, esfenvarelato e fenpropathrin (0,075 g i.a.L $\left.\mathrm{L}^{-1}\right)$ em ovos de Chrysoperla externa (Hagen) (Neuroptera: Chrysopidae), e aos de Moraes \& Carvalho (1993) que ao realizarem pulverizações de fenpropathrin nas doses de 0,4 e 0,12 g i.a.L $L^{-1}$ em ovos de $C$. cubana registraram viabilidades de $81,3 \%$ e $90,4 \%$, respectivamente. 
Referente ao desenvolvimento embrionário, todos os acaricidas estudados foram inócuos, apresentando uma variação de 4,7 a 5,2 dias para a eclosão das larvas (Tabela 2). Esses resultados encontram-se dentro do normal de incubação de 5,08 dias encontrado por Venzon \& Carvalho (1992) para C. cubana.

De acordo com a escala de toxicidade da IOBC, todos os compostos testados foram enquadrados na classe 1, sendo considerados inócuos aos ovos desse predador (Tabela 2).

Efeito de acaricidas na mortalidade e longevidade de adultos de C. cubana. Observou-se que triazophos e carbosulfan causaram $100 \%$ de mortalidade para machos e fêmeas do predador, sendo então classificados na classe 4 (nocivos) (Tabela 3). Os piretróides fenpropathrin e bifenthrin, apesar de apresentarem taxas de mortalidade relativamente altas para adultos com médias de $60 \%$ e $55 \%$, respectivamente, foram classificados na classe 2 (levemente nocivos) (Tabela 3). De acordo com Santa-Cecília et al. (1997), fenpropathrin aplicado em uma dosagem maior $\left(0,15 \mathrm{~g}\right.$ i.a. $\left.\mathrm{L}^{-1}\right)$ apresentou $100 \%$ de mortalidade em adultos de C. cubana, sendo classificado como nocivo. Ulhôa et al. (2002) também constataram $100 \%$ de mortalidade, quando aplicaram fenpropathrin $\left(0,09\right.$ g i.a. $\left.\mathrm{L}^{-1}\right)$ em adultos de C. externa.

Os demais acaricidas foram inócuos, demonstrando que adultos de $C$. cubana apresentam níveis variáveis de tolerância e suscetibilidade aos compostos testados. Esses resultados confirmam aqueles obtidos por Godoy et al. (2004a) em que abamectin $\left(0,0054 \mathrm{~g}\right.$ i.a. $\left.\mathrm{L}^{-1}\right)$ e óxido de fenbutatin $\left(0,4 \mathrm{~g}\right.$ i.a. $\left.\mathrm{L}^{-1}\right)$ aplicados em adultos de $C$. externa foram classificados na classe 1 (inócuos) e, também, por Santa-Cecília et al. (1997) que, ao pulverizarem óxido de fenbutatin $\left(0,025 \mathrm{~g}\right.$ i.a. $\left.\mathrm{L}^{-1}\right)$ em adultos de C. cubana verificaram $100 \%$ de sobrevivência.

A classificação um (1) para a maioria dos acaricidas avaliados nesse estudo indica um alto potencial para inclusão dos mesmos em programas de manejo integrado de pragas. Entretanto, a avaliação de seus efeitos sobre outras espécies benéficas deve ser levada em consideração.

Referente à longevidade de adultos, constatou-se que não houve variação significativa entre os produtos testados, tanto para machos quanto para fêmeas. Verificouse também que a longevidade de ambos os sexos foi, de modo geral, semelhante, sendo que, fêmeas e machos apresentaram períodos médios de vida variando de 35,4 a 52,2 dias (Tabela 3). A longevidade observada para $C$. cubana aproximou-se dos resultados encontrados por Venzon \& Carvalho (1992), em estudo da biologia dessa espécie em condições laboratoriais.

Efeito de acaricidas na oviposição de $\boldsymbol{C}$. cubana. Os compostos avaliados não reduziram os períodos de oviposição e efetivo de oviposição, com médias de 20,9 a 33,6 dias e de 13,7 a 29,3 dias, respectivamente. Esses

Tabela 2 - Viabilidade, período de incubação de ovos de C. cubana e grau de toxicidade dos acaricidas testados ${ }^{(1)}$.

\begin{tabular}{lccc}
\hline Tratamento & Viabilidade $(\%)$ & Período embrionário (dias) & Grau de toxicidade $^{*}$ \\
\hline Dicofol & $95,0 \pm 4,41 \mathrm{ab}$ & $4,8 \pm 0,31 \mathrm{~b}$ & 1 \\
Fenpropathrin & $90,7 \pm 3,55 \mathrm{ab}$ & $4,9 \pm 0,37 \mathrm{~b}$ & 1 \\
Bifenthrin & $85,9 \pm 5,98 \mathrm{ab}$ & $4,9 \pm 0,42 \mathrm{ab}$ & 1 \\
Bromopropylate & $97,0 \pm 8,11 \mathrm{a}$ & $4,8 \pm 0,33 \mathrm{~b}$ & 1 \\
Triazophos & $77,9 \pm 4,30 \mathrm{~b}$ & $5,0 \pm 0,45 \mathrm{ab}$ & 1 \\
Tetradifon & $91,3 \pm 4,66 \mathrm{ab}$ & $4,7 \pm 0,39 \mathrm{~b}$ & 1 \\
Clofentezine & $92,8 \pm 5,73 \mathrm{ab}$ & $4,9 \pm 0,35 \mathrm{~b}$ & 1 \\
Flufenoxuron & $76,8 \pm 4,11 \mathrm{~b}$ & $5,2 \pm 0,31 \mathrm{~b}$ & 1 \\
Carbosulfan & $90,8 \pm 4,58 \mathrm{ab}$ & $4,9 \pm 0,40 \mathrm{~b}$ & 1 \\
Abamectin & $88,4 \pm 6,12 \mathrm{ab}$ & $4,9 \pm 0,39 \mathrm{~b}$ & 1 \\
Hexythiazox & $89,0 \pm 4,15 \mathrm{ab}$ & $5,0 \pm 0,41 \mathrm{~b}$ & 1 \\
Cyhexatin & $92,4 \pm 3,50 \mathrm{ab}$ & $5,0 \pm 0,35 \mathrm{~b}$ & 1 \\
Óxido de fenbutatin & $85,2 \pm 4,86 \mathrm{ab}$ & $4,7 \pm 0,38 \mathrm{~b}$ & - \\
Testemunha & $94,5 \pm 5,10 \mathrm{ab}$ & $5,0 \pm 0,45 \mathrm{~b}$ & 1 \\
\hline
\end{tabular}

${ }^{(1)}$ Médias seguidas da mesma letra na coluna não diferem significativamente entre si, pelo teste de Tukey $(\mathrm{P}>0,05)$.

*Escala de Hassan \& Degrande (1996): classe 1 = inócuo (E < 30\% de mortalidade e/ou influência na reprodução do crisopídeo), classe $2=$ levemente nocivo $(30 \% \leq \mathrm{E} \leq 79 \%)$, classe $3=$ moderamente nocivo $(80 \% \leq \mathrm{E} \leq 99 \%)$ e classe $4=$ nocivo $(\mathrm{E}>99 \%)$. 
resultados aproximam-se aos de Venzon \& Carvalho (1992) que estudaram a biologia dessa espécie de crisopídeo alimentada com dieta à base de mistura de lêvedo de cerveja e mel (1:1) em condições de laboratório.

Efeito de acaricidas na capacidade de oviposição. $O$ acaricida flufenoxuron causou redução na produção diária e total de ovos com médias de 4,9 e 120,8, respectivamente em relação à testemunha que apresentou 10,9 e 354,3 ovos, respectivamente (Tabela 4). Esses resultados estão de acordo com os de Ulhôa et al. (2002) que, ao aplicarem triflumuron, com o mesmo modo de ação do flufenoxuron, em fêmeas de $C$. externa constataram redução na capacidade

Tabela 3 - Mortalidade e longevidade de adultos de C. cubana e grau de toxicidade dos acaricidas testados ${ }^{(1)}$.

\begin{tabular}{|c|c|c|c|c|c|}
\hline \multirow{2}{*}{ Tratamento } & \multicolumn{2}{|c|}{ Mortalidade (\%) } & \multicolumn{2}{|c|}{ Longevidade (dias) } & \multirow{2}{*}{$\begin{array}{c}\text { Grau de } \\
\text { toxicidade }\end{array}$} \\
\hline & Machos & Fêmeas & Machos & Fêmeas & \\
\hline Dicofol & $0,0 \pm 0,00 \mathrm{a}$ & $0,0 \pm 0,0 \mathrm{a}$ & $46,0 \pm 4,50 \mathrm{a}$ & $44,0 \pm 3,31 \mathrm{a}$ & 1 \\
\hline Fenpropathrin & $70,0 \pm 4,89 b$ & $50,0 \pm 4,36 b$ & $35,3 \pm 7,70 \mathrm{a}$ & $45,5 \pm 4,40 \mathrm{a}$ & 2 \\
\hline Bifenthrin & $50,0 \pm 3,99 b$ & $60,0 \pm 5,89 b$ & $54,2 \pm 9,8 \mathrm{a}$ & $52,1 \pm 5,72 \mathrm{a}$ & 2 \\
\hline Bromopropylate & $0,0 \pm 0,00 \mathrm{a}$ & $0,0 \pm 0,00 \mathrm{a}$ & $44,6 \pm 5,41 \mathrm{a}$ & $49,4 \pm 4,87 a$ & 1 \\
\hline Triazophos & $100,0 \pm 0,00 \mathrm{c}$ & $100,0 \pm 0,00 \mathrm{c}$ & - & - & 4 \\
\hline Tetradifon & $0,0 \pm 0,00 \mathrm{a}$ & $0,0 \pm 0,00 \mathrm{a}$ & $48,4 \pm 4,44 a$ & $47,8 \pm 3,99 a$ & 1 \\
\hline Clofentezine & $0,0 \pm 0,00 \mathrm{a}$ & $0,0 \pm 0,00 \mathrm{a}$ & $49,6 \pm 5,55 a$ & $48,7 \pm 3,86 a$ & 1 \\
\hline Flufenoxuron & $0,0 \pm 0,00 \mathrm{a}$ & $0,0 \pm 0,00 \mathrm{a}$ & $42,0 \pm 4,47 a$ & $46,7 \pm 4,41 \mathrm{a}$ & 1 \\
\hline Carbosulfan & $100,0 \pm 0,00 \mathrm{c}$ & $100,0 \pm 0,00 \mathrm{c}$ & - & - & 4 \\
\hline Abamectin & $0,0 \pm 0,00 \mathrm{a}$ & $0,0 \pm 0,00 \mathrm{a}$ & $53,0 \pm 7,84 a$ & $41,8 \pm 5,61 \mathrm{a}$ & 1 \\
\hline Hexythiazox & $0,0 \pm 0,00 \mathrm{a}$ & $0,0 \pm 0,00 \mathrm{a}$ & $46,5 \pm 3,59 a$ & $47,1 \pm 4,58 \mathrm{a}$ & 1 \\
\hline Cyhexatin & $0,0 \pm 0,00 \mathrm{a}$ & $0,0 \pm 0,00 \mathrm{a}$ & $54,0 \pm 8,81 \mathrm{a}$ & $48,6 \pm 5,47 a$ & 1 \\
\hline Óxido de fenbutatin & $0,0 \pm 0,00 \mathrm{a}$ & $0,0 \pm 0,00 \mathrm{a}$ & $55,3 \pm 7,75 a$ & $50,4 \pm 5,02 \mathrm{a}$ & 1 \\
\hline Testemunha & $0,0 \pm 0,00 \mathrm{a}$ & $0,0 \pm 0,00 \mathrm{a}$ & $53,9 \pm 8,88 \mathrm{a}$ & $47,6 \pm 4,89 a$ & - \\
\hline
\end{tabular}

${ }^{(1)}$ Médias seguidas da mesma letra na coluna não diferem significativamente entre si, pelo teste de Tukey $(\mathrm{P}>0,05)$.

* Escala de Hassan \& Degrande (1996): classe 1 = inócuo (E < 30\% de mortalidade e/ou influência na reprodução do crisopídeo), classe $2=$ levemente nocivo $(30 \% \leq \mathrm{E} \leq 79 \%)$, classe $3=$ moderamente nocivo $(80 \% \leq \mathrm{E} \leq 99 \%)$ e classe $4=$ nocivo $(\mathrm{E}>99 \%)$.

Tabela 4 - Capacidade de oviposição diária e total, viabilidade e período de incubação de ovos provenientes de fêmeas de C. cubana tratadas com diferentes acaricidas ${ }^{(1)}$.

\begin{tabular}{lcccc}
\hline \multirow{2}{*}{ Tratamento } & \multicolumn{2}{c}{ Capacidade de oviposição (número de ovos) } & Viabilidade (\%) & $\begin{array}{c}\text { Período } \\
\text { Embrionário (dias) }\end{array}$ \\
\cline { 2 - 3 } Dicofol & Diária & Total & & \\
Fenpropathrin & $13,0 \pm 3,22 \mathrm{a}$ & $352,4 \pm 9,98 \mathrm{a}$ & $76,5 \pm 9,89 \mathrm{a}$ & $4,9 \pm 0,95 \mathrm{a}$ \\
Bifenthrin & $8,9 \pm 2,99 \mathrm{abc}$ & $271,0 \pm 8,88 \mathrm{ab}$ & $77,5 \pm 8,45 \mathrm{a}$ & $4,9 \pm 0,87 \mathrm{a}$ \\
Bromopropylate & $8,3 \pm 3,04 \mathrm{abc}$ & $258,2 \pm 7,98 \mathrm{ab}$ & $80,3 \pm 6,45 \mathrm{a}$ & $5,0 \pm 0,99 \mathrm{a}$ \\
Tetradifon & $10,0 \pm 2,99 \mathrm{ab}$ & $303,3 \pm 11,45 \mathrm{ab}$ & $87,2 \pm 7,48 \mathrm{a}$ & $4,7 \pm 0,78 \mathrm{a}$ \\
Clofentezine & $10,7 \pm 3,32 \mathrm{ab}$ & $325,6 \pm 13,33 \mathrm{a}$ & $88,4 \pm 7,52 \mathrm{a}$ & $5,1 \pm 0,85 \mathrm{a}$ \\
Flufenoxuron & $8,4 \pm 2,75 \mathrm{abc}$ & $268,4 \pm 7,23 \mathrm{ab}$ & $92,4 \pm 8,46$ & $4,8 \pm 0,77 \mathrm{a}$ \\
Abamectin & $4,9 \pm 2,01 \mathrm{c}$ & $120,8 \pm 8,65 \mathrm{~b}$ & $0,0 \pm 0,00 \mathrm{~b}$ & $0,0 \pm 0,00 \mathrm{~b}$ \\
Hexythiazox & $11,2 \pm 2,45 \mathrm{a}$ & $28,2 \pm 9,36 \mathrm{ab}$ & $74,8 \pm 9,99 \mathrm{a}$ & $5,2 \pm 0,91 \mathrm{a}$ \\
Cyhexatin & $6,1 \pm 3,94 \mathrm{bc}$ & $184,5 \pm 8,32 \mathrm{ab}$ & $79,9 \pm 6,32 \mathrm{a}$ & $5,0 \pm 0,84 \mathrm{a}$ \\
Óxido de fenbutatin & $10,6 \pm 2,84 \mathrm{ab}$ & $320,6 \pm 9,87 \mathrm{a}$ & $92,4 \pm 8,97 \mathrm{a}$ & $5,0 \pm 0,88 \mathrm{a}$ \\
Testemunha & $10,2 \pm 3,31 \mathrm{ab}$ & $337,2 \pm 8,76 \mathrm{a}$ & $84,0 \pm 7,23 \mathrm{a}$ & $4,9 \pm 0,97 \mathrm{a}$ \\
\hline
\end{tabular}

${ }^{(1)}$ Médias seguidas da mesma letra na coluna não diferem significativamente entre si, pelo teste de Tukey $(P>0,05)$. 
de oviposição, e com os de Bueno \& Freitas (2004) que verificaram diminuição do número de ovos produzidos pelas fêmeas desse crisopídeo quando pulverizados com lufenuron $\left(0,5\right.$ a 2 g i.a. $\left.\mathrm{L}^{-1}\right)$ e, também, com aqueles obtidos por Mattioli et al. (1992) para C. cubana utilizando os produtos flufenoxuron e diflubenzuron.

À exceção de flufenoxuron e hexythiazox, a produção média de ovos, tanto diária quanto total dos demais tratamentos, aproximou-se dos resultados obtidos por Venzon \& Carvalho (1992) em estudo da biologia de fêmeas de $C$. cubana provenientes de larvas alimentadas com ovos de Anagasta kuehniella (Zeller, 1879) (Lepidoptera: Pyralidae).

Viabilidade e período embrionário. Verificou-se que somente flufenoxuron afetou significativamente a viabilidade dos ovos, inibindo completamente a eclosão das larvas (Tabela 4). Inicialmente, os ovos apresentaram um desenvolvimento embrionário aparentemente normal, entretanto a eclosão da larva foi impedida, ocorrendo um escurecimento e murchamento dos mesmos. Godoy et al. (2004b) também constataram redução significativa na viabilidade dos ovos de fêmeas de C. externa tratadas com lufenuron $\left(0,0375\right.$ g i.a. $\left.\mathrm{L}^{-1}\right)$.

Os resultados obtidos com os acaricidas testados neste estudo evidenciaram que na avaliação da toxicidade de um pesticida para uma espécie benéfica, além dos efeitos deletérios sobre os indivíduos atingidos pela aplicação do produto, devem ser considerados, também, os efeitos subletais, principalmente em relação à capacidade reprodutiva desses espécimes.

\section{CONCLUSÕES}

Ovos de Ceraeochrysa cubana são tolerantes a todos os acaricidas estudados.

Flufenoxuron prolonga o período embrionário de C. cubana, mas não reduz a sua viabilidade.

O carbosulfan e triazophos prejudicam a longevidade de C. cubana.

Flufenoxuron reduz a fecundidade das fêmeas, bem como torna todos os ovos inviáveis.

Os acaricidas dicofol, bromopropylate, tetradifon, clofentezine, abamectin, hexythiazox, cyhexatin e óxido de fenbutatin apresentam baixa toxicidade para $C$. cubana $\mathrm{e}$ podem ser recomendados para programas de manejo de ácaros fitófagos visando à preservação dessa espécie de crisopídeo.

\section{REFERÊNCIAS BIBLIOGRÁFICAS}

BUENO, A.F.; FREITAS, S. Effect of the insecticides abamectin and lufenuron on eggs and larvae of
Chrysoperla externa under laboratory conditions. BioControl, v.49, p.277-283, 2004.

CARVALHO, G.A.; CARVALHO, C.F.; SOUZA, B.; ULHÔA, J.L.R. Seletividade de inseticida a Chrysoperla externa (Hagen) (Neuroptera: Chrysopidae). Neotropical Entomology, v.4, p.615-621, 2002.

FOERSTER, A.L. Seletividade de inseticidas a predadores e parasitóides. In: BENTO, S.J.M. Controle biológico no Brasil: parasitóides e predadores. São Paulo: Manole, 2002. p.95-114.

CARVALHO, C.F.; SOUZA, B. Métodos de criação e produção de crisopídeos. In: BUENO, V.H.P. (Ed.). Controle biológico de pragas: produção massal e controle de qualidade. Lavras: UFLA, 2009. p.77-115.

GODOY, M.S.; CARVALHO, G.A.; MORAES, J.C.; MORAES, A.A.; COSME, L.V. Seletividade de seis inseticidas utilizados em citros a pupas e adultos de Chrysoperla externa (Hagen) (Neuroptera: Chrysopidae). Neotropical Entomology, Londrina, v.33, n.3, p.359-364, 2004a.

GODOY, M.S.; CARVALHO, G.A.; MORAES, J.C.; MORAES, A.A.; COSME, L.V. Seletividade de inseticidas utilizados na cultura dos citros para ovos e larvas de Chrysoperla externa (Hagen) (Neuroptera: Chrysopidae). Neotropical Entomology, Londrina, v.33, n.5, p.639-646, 2004b.

GRAVENA, S. Manejo integrado de pragas de citros na atualidade. In: FERNANDES, O.A. Manejo integrado de pragas e nematóides. São Paulo: UNESP, 1990. v.1, p.253.

HASSAN, S.A.; DEGRANDE, P.E. Methods to test the side effects of pesticides on Thrichogramma. In: PARRA, J.R.P.; ZUCCHI, R. (Eds.). Controle biológico com Thrichogramma. Piracicaba: FEALQ, 1996. p.63-74.

MATTIOLI, E.; CARVALHO, C.F.; SALGADO, L.O.

Efeitos de inseticidas e acaricidas sobre ovos, larvas e adultos do predador Ceraeochrysa cubana (Hagen, 1861) (Neuroptera: Chrysopidae) em laboratório. Ciência e Agrotecnologia, Lavras, v.16, p.491-497, 1992.

MORAES, J.C.; CARVALHO, C.F. Seletividade de acaricidas a ovos, larvas e adultos de Ceraeochrysa cubana (Hagen, 1861) (Neuroptera:Chrysopidae). Ciências e Prática, Lavras, v.17, p.388-392, 1993. 
PARRA, J.R.P.; NEGRI, H.O.; PINTO, A.S. Guia ilustrado de pragas e insetos benéficos dos citros. Piracicaba: ESALQ, 2003. 140p.

SANTA-CECILIA, L.V.C.; SOUZA, B.; CARVALHO, C.F. Seletividade de alguns inseticidas/acaricidas aos adultos de Ceraeochrysa cubana (Hagen, 1861) (Neuroptera: Chrysopidae) em laboratório. Pesquisa Agropecuária Brasileira, Brasília, v.32, n.8, p.803-806, 1997.

SOUZA, B.; SANTA-CECILIA, L.V.C.; CARVALHO, C.F. Seletividade de alguns inseticidas e acaricidas a ovos e larvas de Ceraeochrysa cubana (Hagen, 1861) (Neuroptera: Chrysopidae) em laboratório. Pesquisa
Agropecuária Brasileira, Brasília, v.31, n.11, p.775779, 1996.

ULHÔA, J.L.R.; CARVALHO, G.A.; CARVALHO, C.F.; SOUZA, B. Ação de inseticidas recomendados para o controle do curuquerê-do-algodeiro para pupas e adultos de Chrysoperla externa (Hagen, 1861) (Neuroptera: Chrysopidae). Ciência e Agrotecnologia, Lavras, p.1365-1372, 2002. Edição especial.

VENZON, M.; CARVALHO, C.F. Biologia da fase adulta de Ceraeochrysa cubana (Hagen, 1861) (Neuroptera: Chrysopidae) em diferentes dietas e temperaturas. Ciência e Prática, Lavras, v.16, p.315-320, 1992. 